\title{
Fabrication and Optical Properties of Plasmonic Nanocomposite Structures
}

\author{
$\underline{\text { Y. Andreeva }}^{1}$, P. Varlamov ${ }^{1}$, F. Vocanson ${ }^{2}$, N. Destouches ${ }^{2}$, T. Itina ${ }^{2}$ \\ ${ }^{1}$ ITMO University, Laser photonics and optoelectronics department, 49 Kronverksky pr., 197101, Saint \\ Petersburg, Russia \\ ${ }^{2}$ University of Lyon, Laboratoire Hubert Curien, UMR CNRS 5516/UJM, Saint-Etienne 42000, France \\ andreeva.ym@itmo.ru
}

\begin{abstract}
In this work, we demonstrate a single-step laser-assisted fabrication of thin plasmonic nanocomposite thin films. The common processes taking place in Ag:Au: $\mathrm{TiO}_{2}$ thin nanocomposite films under UV laser irradiation are discussed. We describe the main nanostructure formation mechanisms, as well as their effect on the optical properties of the films. The obtained results pave the way for the implementation of such materials in modern photonics and optoelectronics devices, photocatalytic systems, sensing, and SERS due to their unique and well-controlled optical properties.
\end{abstract}

\section{INTRODUCTION}

The interest in different types of nanocomposite materials is constantly growing during the last decades. Combining the properties of plasmonic inclusions and surrounding matrix it is possible to obtain a nanocmposite materials with unique optical, magnetic, chemical, and/or catalytic properties [1]-[4]. This makes them very attractive for photonics devices, sensing, SERS, photocatalysis, and security applications [3]- [6].

Nanocomposite metamaterials and plasmonic materials containing metallic nanoparticles are shown to be obtained by various methods, among which the most common are chemical synthesis, various spraying methods, lithography, etc. [1]-[6]. However, these methods have their drawbacks and challenges. Some of them are time-consuming and polluting and using them it is impossible to manage the optical and physicochemical properties of the material on a micro- and nanoscale during the fabrication process. Another challenge is to fabricate a system or device with different optical and chemical properties on a single substrate. From this point of view, a promising solution is laser irradiation, which provides the possibility of local control over the crystal phase, and chemical composition of the material, as well as the size and spatial distribution of nanoparticles [6]-[8]. Thus, complex system can be realized during one production step with separated areas of various properties.

Various methods of laser synthesis of nanocomposite materials containing silver nanoparticles as well as methods of tuning their optical properties were already studied in details [5], [7]. In this paper, we propose to use two types of plasmonic nanoparticles ( $\mathrm{Ag}$ and $\mathrm{Au}$ ), which will potentially widely expand the application field of such nanocomposite films. Here we describe the $\mathrm{CW}$ laser radiation interaction with an $\mathrm{Ag}: \mathrm{Au}: \mathrm{TiO}_{2}$ nanocomposite and study the properties of the obtained material plasmonic nanocomposite.

\section{RESULTS AND DISCUSSION}

\section{A. Samples preparation and examination}

Thin nanocomposite films containing silver and gold nanoparticles were prepared for further investigation. For this, the multiple-step process applied. First, mesoporous films of titanium dioxide were soaked in ammonia silver nitrate solution $\left.\mathrm{Ag}\left(\mathrm{NH}_{3}\right)_{2}\right]^{+} \mathrm{NO}_{3}{ }^{-}$during 30 minutes. Then, the film rinsed in ultrapure water, dried with nitrogen, and irradiated under the UV lamp $(254 \mathrm{~nm})$ for 15 minutes to initiate the reduction of silver ions. After that, the film was additionally soaked in $\mathrm{HAuCl}_{4}$ aqueous solution during 1 minute, and chemically reduced in $\mathrm{NaBH}_{4}$. To obtain a uniform distribution of the initial particles in the film, films were dried in dark for 24 hours.

For the formation of $\mathrm{Ag}: \mathrm{Au}: \mathrm{TiO}_{2}$ nanocomposite material and modification of its optical properties the continuous wave (CW) laser (Coherent Innova 70C spectrum) with a wavelength of $488 \mathrm{~nm}$ was used. The choice 
of the source is primarily due to the absorption of the initial film in this region of the spectrum. The beam was focused on the top of the film in a spot of $17 \mathrm{~nm}$ diameter with $20 \times$ microlens. Scanning was carried out by 2-axes coordinate table with servomotors.

The obtained material was studied by scanning electron microscopy using FEI Nova nano SEM 200 model equipped by helix detector. UV-visible transmission spectra were measured with a Cary 5000 spectrophotometer (Agilent).

\section{B. Laser-induced formation of $\mathrm{Ag}: \mathrm{Au}: \mathrm{TiO}_{2}$ plasmonic nanocomposite}

Depending on the laser irradiation power, spot size, and scanning speed, the size, and space distribution of nanoparticles in the composite film were changed. For all laser treatment regimes, the formation of large particles with sizes up to several micrometers at the periphery of the laser track was observed, while nanoparticles with sizes from 10 to $100 \mathrm{~nm}$ predominated in the center of the exposure zone. In line-by-line scanning mode, this effect led to the self-organization of large particles in the form of a fairly well ordered lattice with a period of the scanning step (displacement between two lines along an axis perpendicular to the scanning direction) (see Fig. 1 $\mathrm{a}, \mathrm{b}$ ). For the increased overlap between laser tracks slight self-organization of small nanoparticles along laser polarization was obtained in the central region (insert in 1b). This effect is related to the waveguide mode in the thin film.

It is worth noting that after laser processing, the optical properties of the material changed significantly. The absorption picks intensified due to the redistribution of the nanoparticles' sizes along the track width. In particular when the laser radiation power was in the range of 170-200 $\mathrm{mW}$, the absorption peak blue-shifted with an increase of the scanning speed. As it is demonstrated in Fig. 1c, at a power of $170 \mathrm{~mW}$, the peak position was changed from $518 \mathrm{~nm}$ (at a scanning speed of $500 \mu \mathrm{m} / \mathrm{s}$ ) to $490 \mathrm{~nm}$ (at a scanning speed of 30,000 $\mu \mathrm{m} / \mathrm{s}$ ). On the other hand, when the radiation power was above $200 \mathrm{~mW}$, the dependence of the absorption peak position on the scanning speed became nonlinear (Fig. 1d).

a.

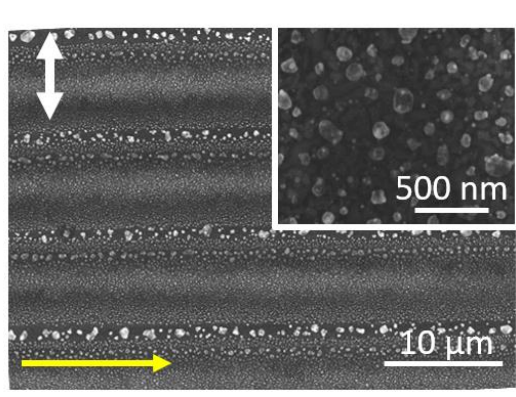

C.

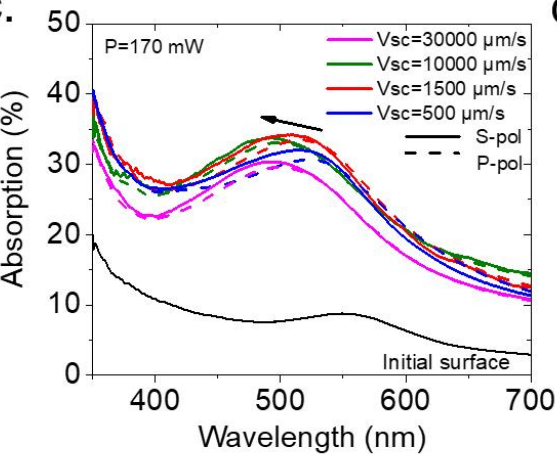

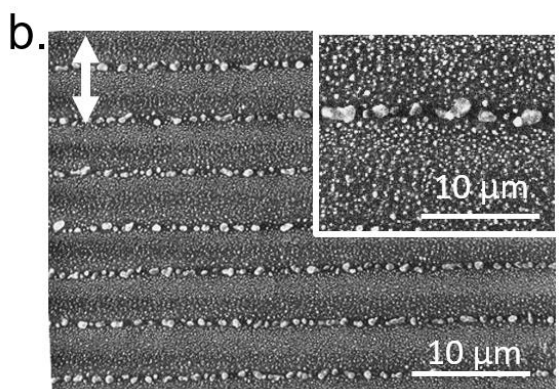

d.

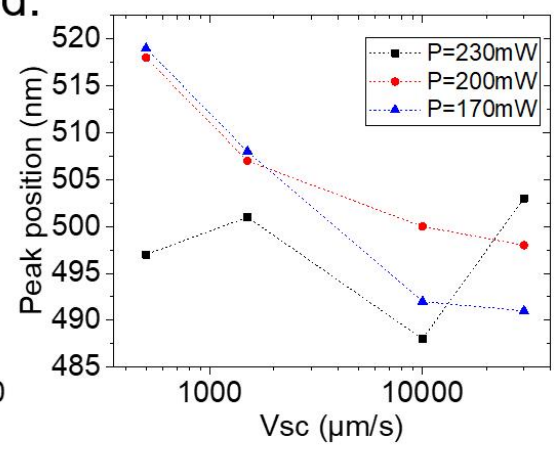

Fig. 1. Characterization of obtained plasmonic nanocomposite system. (a, b) SEM images of nanocomposite films after laser irradiation (laser power $\mathrm{P}=170 \mathrm{~mW}$, scanning speed $\mathrm{V}_{\mathrm{sc}}=30000 \mu \mathrm{m} / \mathrm{s}$ ) recorded with the various overlap between tracks (white double arrow represents laser polarization direction, yellow arrow shows the scanning direction): (a) overlap $=50 \%$; (b) overlap 75\%. (c) absorption coefficient of $\mathrm{Ag}: \mathrm{Au}: \mathrm{TiO}_{2}$ nanocomposite $\left(\mathrm{P}=170 \mu \mathrm{mW}, \mathrm{V}_{\mathrm{sc}}=30000 \mu \mathrm{m} / \mathrm{s}\right.$, overlap $\left.=50 \%\right)$. (d) Dependence of absorption peak position on scanning speed for different laser power (overlap $=50 \%$ ). 
The movement of the absorption peak into the violet region is associated with a decrease of the size of the nanoparticles in the center region of laser track with an increase in the scanning speed. The formation of small particles in the center and much larger particles at the boundaries of the laser track is probably due to the formed temperature field distribution and its influence both on the diffusion growth and coagulation of nanoparticles, and on reverse processes of photooxidation of silver particles. Since the temperature is lower on the periphery, growth processes prevailed there.

\section{CONCLUSION}

Here we briefly discussed the method of laser-assisted single-step fabrication and optical properties of the $\mathrm{TiO}_{2}$-based nanocomposite plasmonic system. Experimentally we identified the common dependences of nanoparticles growth and distribution under UV CW laser irradiation. The reported results allow one to obtain a material with a given size and spatial distribution of nanoparticles, as well as to local control over the absorption of nanocomposite films. In prospect, such systems consisting of separated units with different optical and chemical properties will be interesting for the creation of photocatalytic devices and photoelectric sensors, as well as photonics devices.

\section{ACKNOWLEDGEMENT}

The reported study was funded by RFBR, project number 19-32-90247.

\section{REFERENCES}

[1] B. Deng, R. Xu, K. Zhao, Y. Lu, S. Ganguli, G. J. Cheng, "Composite bending-dominated hollow nanolattices: A stiff, cyclable mechanical metamaterial," Mater. Today, vol. 21(5), pp. 467-474, 2018.

[2] H. Cui, R. Hensleigh, D. Yao, D. Maurya, P. Kumar, M. G. Kang, S. Priya, X. R. Zheng, "Three-dimensional printing of piezoelectric materials with designed anisotropy and directional response," Nature Mater. vol.18, pp. 234-241, 2019.

[3] H. O. Everitt, T. Tyler, B. D. Caraway, C.M. Bingham, A. Llopis, M. S. Heimbeck, W. J. Padilla, D. R. Smith, N. M. Jokerst, "Strain sensing with metamaterial composites," Adv. Opt. Mater., vol. 7(9), p. 1801397, 2019.

[4] N. Bai, J. Shen, C. Shen, X. Sun, P. Pan, C. Jun, J. Feng, "A broadband composite complementary double- split rectangular metamaterial absorber for planar microwave circuits," Microw. Opt. Technol. Lett, vol. 61(11), pp. 2614-2618, 2019.

[5] M. Beresna, P. G. Kazansky, O. Deparis, I. C. Carvalho, S. Takahashi, A. V. Zayats, "Poling- Assisted Fabrication of Plasmonic Nanocomposite Devices in Glass," Adv. Mater., vol. 22(39), pp. 4368-4372, 2010.

[6] N. Sharma, N. Destouches, C. Florian, R. Serna, J. Siegel, "Tailoring metal-dielectric nanocomposite materials with ultrashort laser pulses for dichroic color control," Nanoscale, vol. 11(40), pp. 18779-18789, 2019.

[7] J. Wei, Z. Zang, Y. Zhang, M. Wang, J. Du, X. Tang, "Enhanced performance of light-controlled conductive switching in hybrid cuprous oxide/reduced graphene oxide ( $\mathrm{Cu} 2 \mathrm{O} / \mathrm{rGO})$ nanocomposites," Opt. letters, vol. 42(5), pp. 911-914, 2017.

[8] N. Sharma, H. Ma, T. Bottein, M. Bugnet, F. Vocanson, D. Grosso, T. E. Itina, Y. Ouerdane, N. Destouches, "Crystal Growth in Mesoporous TiO2 Optical Thin Films," J. Phys. Chem. C, vol. 23(10), pp. 6070-6079, 2019. 